\title{
Determinantes DEL CONTROL INTERNO EN LA GESTIÓN DEL CRÉDITO DE LAS COOPERATIVAS DEL ECUADOR
}

\author{
DETERMINANTS OF INTERNAL \\ CONTROL IN THE CREDIT \\ MANAGEMENT OF ECUADORIAN \\ COOPERATIVES
}

\author{
Verónica Natalia Espinoza Farfán ${ }^{1}$ \\ Juan Pablo Vázquez Loaiza ${ }^{2}$
}

1 Doctora en Contaduría de la Universidad Autónoma de Nuevo León. Magíster en Auditoría Integral de la Universidad Técnica Particular de Loja. Docente de la Carrera de Contabilidad y Auditoría de la Universidad Politécnica Salesiana. Cuenca, Ecuador. Correo electrónico: vespinoza@ups.edu.ec

Orcid: https://orcid.org/0000-0003-4322-4365

2 Doctor en Proyectos de la Universidad Internacional Iberoamericana de México. Investigador y miembro del Grupo de Investigación para la Gestión de las MiPymes de la Universidad Politécnica Salesiana. Cuenca, Ecuador. Correo electrónico: jvazquez@ ups.edu.ec

Orcid: https://orcid.org/0000-0003-2253-9368

Código JEL: M42

Fecha de recepción: 23/01/2020

Fecha de aceptación: 11/05/2020

DOI: https://doi.org/10.18601/16577175.n27.06 


\section{RESUMEN}

Este artículo presenta los factores de control interno que impactan positivamente en la gestión del crédito de las cooperativas de ahorro y crédito del segmento 1 del Ecuador, con información primaria obtenida tras la aplicación de un instrumento de medición mediante escala de Likert a la muestra seleccionada, la misma que es tratada con el modelo de Logit ordenado procurando encontrar la relación entre las variables que intervienen en la investigación. Los resultados muestran que los factores de control interno relacionados con el ambiente de control, la evaluación de riesgo, las actividades de control y la información y comunicación desempeñan un papel muy importante para predecir la probabilidad de que estas instituciones cuenten con una buena gestión del crédito, mientras que el factor de control interno concerniente a la supervisión y monitoreo tiene un impacto no significativo debido a que ya se encuentra inherente en la operatividad de los otros factores.

Palabras clave: cooperativas; control interno; gestión del crédito.

\section{Abstract}

This article presents the internal control factors that positively impact the credit management of the Savings and Credit Cooperatives of Segment 1 of Ecuador, with primary information obtained after the application of a measurement instrument using the Likert scale to the selected sample, the same that is treated with the Logit model ordered trying to find the relationship between the variables involved in the investigation. The results show that internal control factors related to the control environment, risk assessment, control activities and information and communication have a very important role in predicting the probability that these institutions have good credit management, while the internal control factor concerning supervision and monitoring has a non-significant impact because it is already inherent in the operability of the other factors.

Keywords: internal control; credit management; cooperatives.

\section{INTRODUCCIÓN}

Actualmente el cooperativismo es mundialmente conocido y aceptado en los diferentes contextos socioeconómicos ya que tiene una activa participación en el desarrollo de los países debido a que desempeña un papel trascendente en el combate a la marginalidad, la pobreza y la injusta distribución de la riqueza, dado que aporta al fortalecimiento de la democracia; por tal motivo, la Asamblea General de las Naciones Unidas declaró el 2012 el Año Internacional de las Cooperativas (onu, 2011).

En este aspecto, Miño (2013) señala que el cooperativismo en el Ecuador es importante porque surge como una iniciativa que engloba a amplios sectores sociales, 
razón por la cual los cambios actuales, la evolución económica y la estrategia empresarial al servicio de la toma de decisiones exigen el fortalecimiento del sistema de control interno para este tipo de organizaciones, en un claro sentido de complemento y apoyo a la labor gerencial.

En el mismo contexto, Levin y Verbeke (2002) argumentan que la construcción de mecanismos que potencien la investigación y las prácticas cooperativas son factores claves para el fortalecimiento del sector cooperativo con base en necesidades detectadas e investigadas, ya que permitirá cumplir el propósito de ambos y la investigación pasará a ser estable y permanente. Siendo así, esta perspectiva busca aportar en este trabajo una aproximación relacional que precisamente favorezca la gestión de estas instituciones en consideración del espíritu y la filosofía social que, sobre su constitución, han sido formados.

En este sentido, analizar los factores de control interno que impactan en la gestión del crédito de las cooperativas de ahorro y crédito (COAC) del segmento $1^{3}$ del Ecuador resulta oportuno, ya que beneficiará a estas organizaciones del sector financiero popular y solidario al proponer estrategias que permitan hacer más eficiente su funcionalidad. En otras palabras, los resultados obtenidos en el presente estudio permiten responder la interrogante de cuáles son los factores de control interno que impactan en la gestión del crédito de estas instituciones, $y$, al mismo tiempo, aportan para la comprobación de la hipótesis relacionada con que los factores de control interno impactan significativamente en la gestión del crédito de las coAC del segmento 1 del Ecuador.

En este segmento del Ecuador, los estudios sobre los factores de control interno que impactan en la gestión del crédito son pocos, ya que la mayoría se enfoca en las pequeñas, medianas o grandes empresas dejando a un lado a las cooperativas de ahorro y crédito y específicamente al proceso de la gestión del crédito (Dávila, 2003; Da Ros, 2007; Castańeda, 2014; González, 2014; Betancourt, 2015; Díaz \& Tubón, 2018). De ahí el interés por hacer una aportación a la investigación acerca del tema; para lo que se aplica un instrumento de medición a través de la escala de Likert que permite obtener información de la muestra seleccionada y definir los componentes a ser tratados en la investigación por medio de la aplicación del modelo Logit ordenado.

El presente estudio se organiza como sigue: inicia con una revisión de la literatura relacionada con los factores de control interno y de la reglamentación y funcionamiento de las cooperativas de ahorro y crédito del segmento 1 del Ecuador; posteriormente se aplica la metodología definida para la obtención y tratamiento de la información, con lo que se procede a analizar y discutir los resultados obtenidos en la aplicación del modelo, para finalmente emitir las respectivas conclusiones y presentar recomendaciones o sugerencias en relación con el tema de investigación.

3 El segmento 1 se encuentra conformado por las cooperativas de ahorro y crédito que superan los 80 millones de dólares del total de sus activos, según resolución expedida por la Junta de Política y Regulación Monetaria y Financiera (JPRMF, 2015). 


\section{REVISIÓN DE LA LITERATURA}

A nivel mundial, el cooperativismo nace como una respuesta válida ante las carencias del pueblo como una ayuda a los pequeños comerciantes, industriales y artesanos campesinos, en busca de su bienestar y mejora de sus condiciones de vida. Está íntimamente involucrado con la economía social, ya que se basa en una serie de principios y valores que están de igual manera direccionados con este pensamiento económico (Chaves y Monzón, 2001) y representan la columna vertebral de esta economía (Monzón, 1996 citado en Castro, 2015). De igual manera, el cooperativismo arrancó formalmente en el Ecuador a inicios del siglo $\mathrm{xx}$, con el nacimiento de varias cooperativas de consumo y ahorro y crédito promovidas por el Estado, las organizaciones religiosas, los patronos y la cooperación internacional (Miño, 2013).

Por otro lado, al hacer referencia específicamente al origen de las cooperativas de ahorro y crédito a nivel mundial se menciona que su nacimiento reflejó un gran crecimiento después de la experiencia de los pioneros de Rochdale ${ }^{4}$ y se conformaron dos grandes sistemas en Alemania (Miño, 2013). Al mismo tiempo, considerando el origen de dichas instituciones en el Ecuador, la Constitución de la República promulgada en el ańo 2008 reconoce por primera vez un sistema económico social y solidario centrado en el ser humano, de donde el artículo 309 señala que el sistema financiero nacional se compone de los sectores público, privado y del popular y solidario; y particularmente el artículo 311 señala que el sector financiero popular y solidario se compondrá de cooperativas de ahorro y crédito, entidades asociativas o solidarias, cajas y bancos comunales, cajas de ahorro y que las iniciativas de servicios de ese sector recibirán un tratamiento diferenciado y preferencial del Estado, en la medida en que impulsen el desarrollo de la economía popular y solidaria.

Es preciso señalar que estas organizaciones son creadas por el aporte y la asociación voluntaria de personas de bajos ingresos, especialmente del sector rural, que buscan incorporar la responsabilidad social dentro de su gestión; estas personas se constituyen con personería jurídica, disponen de autonomía propia, con capital conformado por la participación de sus socios fundadores (Mayorga y Llagua, 2018).

De acuerdo con la revisión de las investigaciones aplicadas, se encuentran varios estudios (Viloria, 2005; Ablan y Méndez, 2010; Castañeda, 2014) en donde los componentes del control interno basados en el informe Committe of Sponsoring Organizations of the Treadway Commission (coso) han tenido un impacto positivo en el fortalecimiento de la operatividad de las organizaciones. Si se revisan los procesos que se desarrollan en las organizaciones se encuentra que cada vez más se requiere de una gestión adecuada, oportuna, efectiva y en tiempo real que apoye y garantice la toma de decisiones (Alba, 2019).

4 La Sociedad Equitativa de los Pioneros de Rochdale, fundada en 1844 en Inglaterra, fue una cooperativa de consumo y la primera en distribuir entre sus socios los excedentes generados por la actividad, formando las bases del movimiento cooperativo moderno. 
Cabe mencionar que es preciso considerar al marco normativo de referencia internacional relacionado con el control interno, el mismo que seńala al Marco Integrado de Control Interno o comúnmente conocido como Informe coso que fue desarrollado originalmente en el año de 1992 por el Committe of Sponsoring Organizations of the Treadway Commission (coso) y es el resultado de la investigación de un grupo de trabajo integrado por una diversidad de agrupaciones profesionales de alto renombre a nivel mundial, con el objetivo de establecer y definir un marco conceptual de control interno capaz de integrar el sinnúmero de definiciones y conceptos existentes hasta el momento.

Así, por ejemplo, Castañeda (2014) establece que las organizaciones deben establecer un mínimo de reglas de operatividad para lograr sus objetivos, que se denomina sistema de control interno, el cual está sujeto a regulaciones nacionales e internacionales, pero cuya puesta en marcha y óptimo funcionamiento es responsabilidad de la administración de cada organización. De igual manera, Viloria (2005) manifiesta que las organizaciones, para lograr sus objetivos, deben establecer un mínimo de reglas de operatividad, a las que se puede denominar "control interno".

Por su parte, González (2014) argumenta que la relevancia de poseer un sistema de control interno para las organizaciones radica en que a través de este se organizan los procesos de la institución y se orientan hacia la satisfacción de las necesidades que exhiba en un momento determinado, se protegen todos aquellos activos sociales que hacen parte de la estructura patrimonial de la empresa y se contrasta la eficiencia y la efectividad de las operaciones desarrolladas. En este contexto, se erige el sistema de control interno como mecanismo que sustenta la consecución de los objetivos del plan estratégico y la misión y visión institucional, a través de la implementación de métodos sistemáticos orientados a la prevención, el autocontrol y la medición del grado de cumplimiento de las finalidades establecidas (Mejía, 2002).

El Comité de Contadores Profesionales en Empresas (PAIB) de la Federación Internacional de Contadores (IFAC), con la colaboración del Comité de Organizaciones Patrocinantes del coso (2011), lanzó una encuesta para investigar lo necesarias e importantes que son las prácticas de gestión de riesgo y control interno en las empresas de América Latina, llegando a las siguientes conclusiones: el 60\% de las organizaciones cuenta con un sistema oficial tanto de gestión de riesgo como de control interno, el 8\% cuenta con un sistema de gestión de riesgo, el 18\% con uno de control interno y el $14 \%$ no cuenta con ninguno de los dos sistemas.

Y, por su parte, Saromo y Pacheco (2014) establecen que estos resultados cuantitativos permiten afirmar que más de la mitad de las empresas privadas latinoamericanas tiene implementado un sistema de control interno; sin embargo, por ser el único estudio que demuestra cuantitativamente la proporción de empresas privadas que tienen control interno no se puede afirmar categóricamente que en Latinoamérica más de la mitad de las empresas privadas lo tienen implementado.

Mientras que Vélez (2011) sostiene que la causa principal que dio origen al control interno en México fue la gran empresa, como consecuencia del notable aumento de la producción, los propietarios de los negocios se vieron imposibilitados 
de continuar atendiendo personalmente los problemas productivos, comerciales y administrativos, viéndose forzados a delegar funciones dentro de la organización, creando e implementando sistemas de control interno, que sirvan como herramienta de gestión, para prevenir y disminuir fraudes y errores.

En lo que concierne a Viloria (2005) en su investigación llevada a cabo en el municipio Libertador del estado de Mérida en Venezuela, tuvo como resultado fundamental el hecho de que las empresas no diseñan el control interno bajo la óptica de sistemas, y no toman en cuenta los factores recomendados por el informe coso, ya que hacen énfasis en la estructura de la organización y el control de ciertas áreas operativas, sin visualizar al sistema de control interno de forma integral.

De la misma forma, Ablan y Méndez (2010) realizaron un estudio para analizar los sistemas de control interno de los entes descentralizados estatales y municipales adscritos al municipio Libertador y a la gobernación del estado de Mérida en Venezuela, concluyendo que estas instituciones perciben el control interno como un requisito legal y no como un proceso sistémico para la mejora continua de la organización.

Castañeda (2014) llevó a cabo un estudio comparativo con tres empresas del sector confección de la ciudad de Medellín, en Colombia, una mediana, una pequeña y una micro, con el fin de precisar la contribución del sistema de control interno al logro de los objetivos de estas organizaciones utilizando como referencia el modelo coso. El resultado obtenido demuestra un sistema de control interno deficiente, debido a que los controles se fundamentan en la experiencia empírica de quienes las administran y dirigen, y no poseen el carácter metodológico y formal para llegar a la evaluación permanente del cumplimiento de los objetivos organizacionales. En este trabajo se identificó cómo las micro, pequeñas y medianas empresas (mipymes) pueden pasar de un estado de informalidad y flexibilidad en los procesos de planificación y control hasta una fase de mayor formalidad, con el establecimiento de procesos y procedimientos. De acuerdo con la investigación, el modelo coso puede utilizarse como una herramienta de apoyo a la gestión organizacional, independiente del tamaño de la empresa y contribuye estratégica y operativamente al logro de los objetivos misionales.

Y, finalmente, Dorta (2005) afirma que esta visión sistémica del control interno aporta numerosas posibilidades a la hora de evaluarlo, como que el control interno es fundamental para todas las actividades y unidades de la organización o para la empresa en general, y así segmentar la evaluación por unidades departamentales, por áreas o por actividades funcionales.

Así, en general, el control interno busca la eficiencia y eficacia, sin embargo, no importa qué tan bien ha sido concebido y operado, puede proveer solamente seguridad razonable — no absoluta- a la administración y a la junta directiva mirando la consecución de los objetivos de la cooperativa, pero no asegura el éxito ni supervivencia. En consecuencia, la administración de las cooperativas dependerá del control que se realice, por eso el éxito o fracaso está estrechamente relacionado con una buena o mala administración, esto es más importante en las cooperativas 
por tener una organización y una vida económica encaminada para prestar buenos servicios a sus socios y a la comunidad en general (Trigo, 2012).

Por tal motivo, las cooperativas de ahorro y crédito han demostrado tener un potencial de crecimiento - representan una parte importante de los sistemas no bancarios_- sin embargo, requieren mejorar en eficiencia y rentabilidad, con el fin de garantizar su continuidad (Zaldívar y Sotomayor, 2003).

De tal manera, entonces, que el autocontrol es un principio cooperativo de gran valor agregado para una cooperativa de ahorro y crédito, puesto que permite el conocimiento profundo de la institución y facilita de esta manera la gestión de esta. El sistema de control interno pone a prueba la esencia de este principio en las cooperativas de ahorro y crédito, puesto que, de su efectividad depende el cumplimento de los objetivos institucionales (Cabezas, 2006). En este sentido, el control interno es el principal pilar de un autocontrol adecuado de una coAc. La calidad del control interno es decisiva para el desempeño de la entidad y servirá de base para el trabajo que deba realizar el auditor externo y el órgano supervisor (Arzbach, Durán y Báez, 2010).

En Ecuador, la decisión de las organizaciones para superar los obstáculos en la consolidación del modelo cooperativo se ve reflejada en las experiencias de trabajo de algunas cooperativas que han logrado crecer y producir desarrollos locales, con la ayuda de aquellas que tienen objetivos de canalizar recursos financieros (Gutiérrez, 2009).

En este sentido, la importancia que está adquiriendo el control interno en el último tiempo, a causa de numerosos problemas producidos por su ineficiencia, ha hecho necesario que los miembros de los consejos de administración estén obligados a asumir de forma efectiva una responsabilidad que hasta ahora se habían dejado en manos de todos, lo que claramente significa en manos de nadie. Por eso es preciso que la administración tenga claro en qué consiste el control interno para que pueda actuar y no se originen problemas de comunicación y diversidad de expectativas, que den origen a problemas dentro de las empresas. Al asumir la administración esta responsabilidad por el control interno se incorporan reales beneficios, para el propio sistema de control interno, y en definitiva para toda la organización, debido a que se mejora de manera sustancial el ambiente de control, la evaluación de los riesgos, las actividades de control, la información y comunicación, como también la supervisión (Orellana, Gaete y Gaete, 2002).

Cabe mencionar que no hay suficiente investigación relacionada con el tema bajo estudio. De ahí el interés de constituir una línea con un alto potencial de desarrollo científico y conocimiento que contribuya a potenciar este sector relevante de la economía social (Castro, 2015). En lo referente a los trabajos científicos publicados hasta la fecha, han consolidado unas determinadas líneas de investigación, como el comportamiento estratégico, el estudio de la integración-concentración y el análisis de la rentabilidad, eficiencia y productividad, dentro del ámbito del crédito cooperativo; pero están surgiendo nuevas líneas de investigación con una perspectiva integradora y adaptadas a las nuevas tendencias de la economía (Palacio, 2006). 
La presente investigación es un primer paso para crear teoría propia y proponer soluciones concretas, sin negar otras perspectivas y enfoques. Es así como en este estudio se considera a Castañeda (2014) para determinar que la efectividad del control interno se mide con el transcurso del tiempo y se determina con un juicio que resulta de evaluar si los cinco componentes se dan y funcionan con una alta seguridad, lo cual es el resultado de la consecución de una o varias de las categorías establecidas.

\section{Metodología}

El tipo de investigación es un enfoque mixto, considerando en principio el aspecto cuantitativo, toda vez que se elabora un instrumento de medición que permite obtener información a través de la escala Likert y, por otra parte, se establece el presente estudio con características de tipo cualitativo considerando la aplicación de entrevistas a expertos. Así como también se realizó bajo un esquema de investigación no experimental y se utilizaron diversas técnicas de investigación como procedimientos complementarios al método científico, tales como: la técnica documental, la bibliográfica y la de campo.

Se inició aplicando un estudio exploratorio debido a que la revisión de la literatura existente reveló la escasez de antecedentes sobre el tema en cuestión; también se utilizó el estudio descriptivo para establecer las principales características del fenómeno estudiado.

La presente investigación analizó los factores de control interno que impactan en la gestión del crédito de las cooperativas de ahorro y crédito del segmento 1 del Ecuador (véase la figura 1); es por ello por lo que la unidad de análisis estuvo constituida por 30 instituciones financieras de acuerdo con la distribución de las entidades del sector financiero popular y solidario determinada por la Junta de Política y Regulación Monetaria y Financiera.

Conforme a lo dispuesto en el artículo 14, numeral 35, del Código Orgánico Monetario y Financiero en el que determina entre las funciones de la Junta de Política y Regulación Monetaria y Financiera el establecer la segmentación de las entidades del sector financiero popular y solidario, se expide la resolución 038-2015-F el 13 de febrero de 2015, en la que establece la norma para la segmentación de las entidades del sector financiero popular y solidario (véase tabla 1 ).

En este sentido, el segmento 1 para el año 2018 se encuentra conformado por 30 cooperativas de ahorro y crédito, de las cuales la población abarca un total de 150 actores - tanto funcionarios como directivos — incluyendo a la gerencia general, al consejo de administración, al consejo de vigilancia, al área de créditos y a auditoría interna, considerados de la siguiente manera:

N. ${ }^{\circ}$ de $\operatorname{cooperativas~}^{*}$ N. ${ }^{\circ}$ de sujetos de estudio por cooperativa = Población total

$$
30 * 5=150
$$


Figura 1. Distribución de cooperativas financieras por tipo de segmento
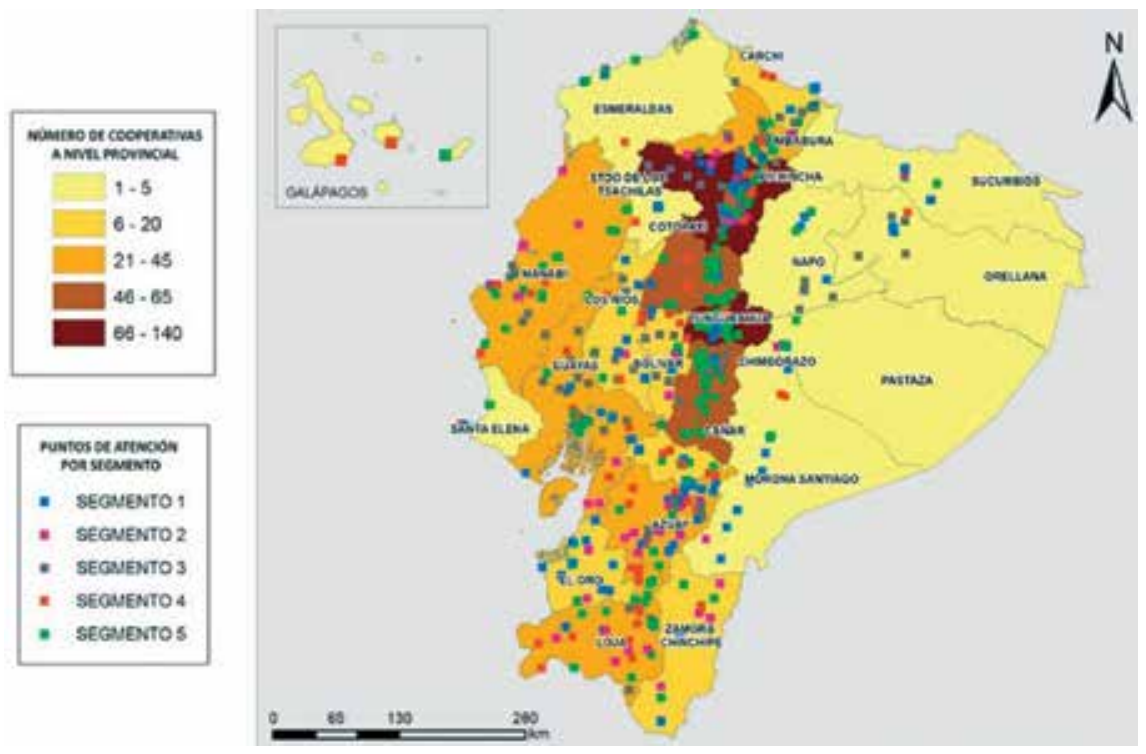

Fuente: Dirección Nacional de Información Técnica y Estadísticas, 2018.

Tabla 1. Segmentación de las entidades del sector financiero popular y solidario de Ecuador

\begin{tabular}{|c|l|}
\hline Segmento & \multicolumn{1}{c|}{ Activos (USD) } \\
\hline 1 & Mayor a 80.000.000,00 \\
\hline 2 & Mayor a 20.000.000,00 hasta 80.000.000,00 \\
\hline 3 & Mayor a 5.000.000,00 hasta 20.000.000,00 \\
\hline 4 & Mayor a 1.000.000,00 hasta 5.000.000,00 \\
\hline \multirow{2}{*}{5} & Hasta 1.000.000,00 \\
\cline { 2 - 2 } & Cajas de ahorro, bancos y cajas comunales \\
\hline
\end{tabular}

Fuente: Resolución 038-2015-F, 13 de febrero de 2015, de la Junta de Política y Regulación Monetaria y Financiera. Elaborado por: Dirección Nacional de Estadísticas y Estudios de la EPs y sfrs.

De tal manera que considerando la población definida con anterioridad y utilizando la fórmula para calcular el tamaño de la muestra resultaron 108 encuestas que aplicar. Por tal motivo, se diseñó un cuestionario con la escala de Likert para conocer el nivel de impacto que tiene el control interno en el objeto de estudio, el mismo que se valida utilizando el método de jueces expertos y aplicando el Alfa de Cronbach en la respectiva prueba piloto. Con los ajustes respectivos se cuenta con el instrumento 
de medición adecuado que se aplicó en la muestra seleccionada obteniendo los datos que permitieron definir los componentes a ser tratados en la presente investigación por medio de la aplicación del modelo Logit ordenado.

Por su parte, al analizar los factores de control interno que impactan en la gestión del crédito de estas instituciones financieras se constituyen como las variables independientes, mientras que la variable dependiente hace referencia a la gestión del crédito, tal cual se presenta en la figura 2 .

Figura 2. Modelo gráfico de la hipótesis

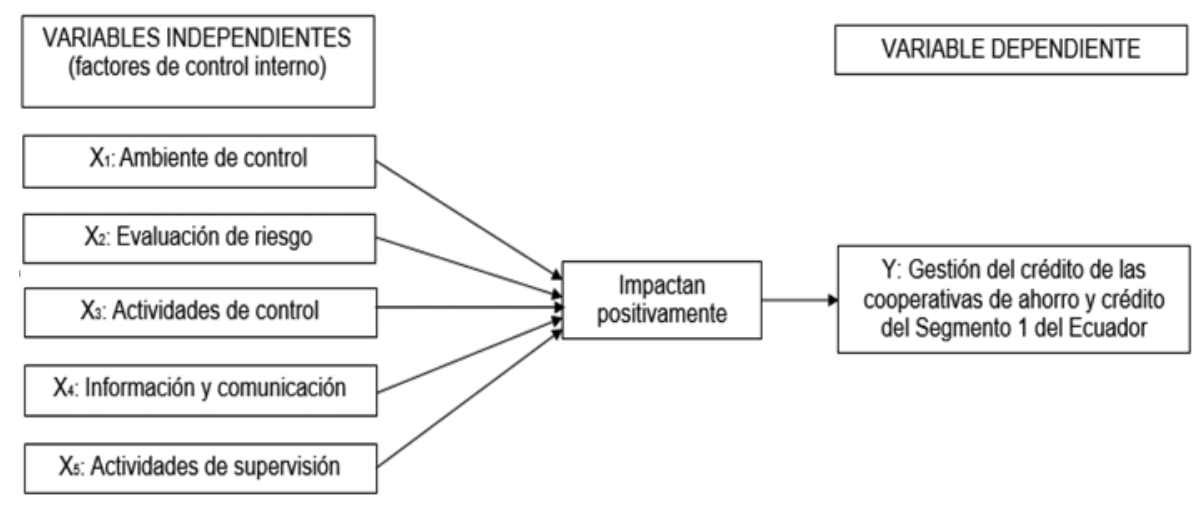

Fuente: elaboración propia.

\section{RESULTADOS Y DISCUSIÓN}

En primera instancia se realizó una tabulación transpuesta de los datos como requisito necesario para ser incluidos en el software estadístico Statistical Package for the Social Sciences (sPss), en donde se dio inicio al tratamiento de la información recolectada obteniendo los estadísticos descriptivos que presentan en promedio general una desviación estándar considerablemente alta para la escala, como lo muestra la tabla 2.

Una vez definidos los componentes se dispone del insumo necesario para proceder con el análisis de la correlación entre las variables, para lo cual se utilizó el software Eviews en la aplicación del modelo Logit. Pérez (2005) señala que para el caso del modelo Logit la función utilizada es logística, por lo que la especificación de este tipo de modelo es como sigue:

$$
\mathrm{Y}_{i}=\frac{1}{1+e^{-a-b_{k} X_{k i}}}+e_{i}=\frac{e^{a+b_{k} X_{k i}}}{1+e^{a+b_{k} X_{k i}}}
$$


Tabla 2. Estadísticos descriptivos

\begin{tabular}{|c|c|c|c|c|c|}
\hline & $\mathbf{N}$ & Mínimo & Máximo & Media & Desviación estándar \\
\hline Proceso formal & 108 & 1 & 5 & 4,37 & 804 \\
\hline Procedimiento crédito & 108 & 2 & 5 & 4,35 & ,727 \\
\hline Política recuperación & 108 & 1 & 5 & 4,37 & ,792 \\
\hline Información confiable & 108 & 1 & 5 & 4,28 & 852 \\
\hline Cumplimiento normativo & 108 & 1 & 5 & 4,10 & 853 \\
\hline Índice morosidad & 108 & 1 & 5 & 4,25 & 968 \\
\hline Código de ética & 108 & 1 & 5 & 3,81 & 826 \\
\hline Valores éticos & 108 & 1 & 5 & 3,90 & ,808 \\
\hline Supervisión funcionamiento & 108 & 1 & 5 & 4,37 & 849 \\
\hline Manual funciones & 108 & 1 & 5 & 4,22 & ,777 \\
\hline Estructura organizacional & 108 & 1 & 5 & 3,99 & 859 \\
\hline Capacitación & 108 & 1 & 5 & 4,41 &, 865 \\
\hline Evaluación desempeño & 108 & 1 & 5 & 4,24 & 852 \\
\hline Plan de incentivos & 108 & 1 & 5 & 4,02 & 967 \\
\hline Objetivos operacionales & 108 & 2 & 5 & 4,18 & ,783 \\
\hline Objetivos crédito & 108 & 1 & 5 & 4,10 & ,937 \\
\hline Mecanismos evaluación & 108 & 1 & 5 & 4,17 & 826 \\
\hline Créditos vinculados & 108 & 1 & 5 & 3,68 & 895 \\
\hline Implementación control & 108 & 1 & 5 & 4,05 & 1,017 \\
\hline Monitoreo & 108 & 1 & 5 & 3,98 & ,785 \\
\hline Segregación funciones & 108 & 1 & 5 & 4,15 & 873 \\
\hline Plan contingencia & 108 & 1 & 5 & 3,97 & 891 \\
\hline Reglamento interno & 108 & 1 & 5 & 4,39 & ,783 \\
\hline Manual procesos & 108 & 1 & 5 & 4,31 & ,803 \\
\hline Actividades control & 108 & 1 & 5 & 4,32 &, 734 \\
\hline Rendición cuentas & 108 & 1 & 5 & 4,01 & ,972 \\
\hline Transformación datos & 108 & 1 & 5 & 4,06 & 889 \\
\hline Utilización fuentes & 108 & 1 & 5 & 4,05 & 890 \\
\hline Comunicación interna & 108 & 1 & 5 & 3,94 & ,777 \\
\hline Evaluaciones externas & 108 & 1 & 5 & 4,02 & ,797 \\
\hline Comunicación terceros & 108 & 1 & 5 & 3,95 & ,790 \\
\hline Canales comunicación & 108 & 1 & 5 & 4,02 & ,864 \\
\hline Logro objetivos & 108 & 1 & 5 & 3,86 & 837 \\
\hline Línea base & 108 & 1 & 5 & 4,21 & ,832 \\
\hline Evaluaciones concurrentes & 108 & 1 & 5 & 3,94 &, 868 \\
\hline Consejo vigilancia & 108 & 1 & 5 & 4,39 & 852 \\
\hline Evaluaciones separadas & 108 & 1 & 5 & 4,12 & 806 \\
\hline Medidas correctivas & 108 & 1 & 5 & 4,48 & 891 \\
\hline Monitoreo correctivo & 108 & 1 & 5 & 4,46 &, 858 \\
\hline $\mathrm{N}$ válido (por lista) & 108 & & & & \\
\hline
\end{tabular}

Fuente: elaboración propia con base en los resultados arrojados por el software spss. 
En este sentido, se aplicó el modelo Logit ordenado debido a las características de la información, principalmente el hecho de que al haberse aplicado una escala de Likert los datos siguieron un orden. Así también, porque la variable dependiente expresó preferencias, opiniones y sobre todo experiencias de los individuos en relación al tema estudiado — gestión del crédito - y las alternativas del proceso de decisión expresaron implícitamente un orden de utilidad y tuvieron por tanto un carácter ordinal, siendo la especificación de este tipo de modelo la siguiente:

$$
\begin{aligned}
& \operatorname{Prob}\left(\mathrm{Y}_{i}=0\right)=\Lambda\left(-\beta^{\prime} \mathrm{X}_{i}\right) \\
& \operatorname{Prob}\left(\mathrm{Y}_{i}=1\right)=\Lambda\left(\mu_{1}-\beta^{\prime} \mathrm{X}_{i}\right)-\Lambda\left(-\beta^{\prime} \mathrm{X}_{i}\right) \\
& \operatorname{Prob}\left(\mathrm{Y}_{i}=2\right)=\Lambda\left(\mu_{2}-\beta^{\prime} \mathrm{X}_{i}\right)-\Lambda\left(\mu_{1}-\beta^{\prime} \mathrm{X}_{i}\right) \\
& \cdots \\
& \operatorname{Prob}\left(\mathrm{Y}_{i}=(J-1)\right)=1-\Lambda\left(\mu_{(J-2)}-\beta^{\prime} \mathrm{X}_{i}\right)
\end{aligned}
$$

Donde $\mu_{1}, \mu_{2} \ldots, \mu_{(J-2)}$ son parámetros que representan los valores de los umbrales o barreras y se estiman a la vez que $\beta$ y $\Lambda\left(\beta^{\prime} \mathrm{X}_{i}\right)$ representa la función de distribución logística.

En este contexto, la inclusión de la información que aportó el orden de las alternativas en la especificación del modelo permitió obtener mejores resultados. Se realizaron varias combinaciones de la ecuación —alternando la exclusión e inclusión de cada una de las variables - de tal manera que se obtuvieron los mejores resultados del modelo, los mismos que se muestran en la tabla 3, donde se presentan las variables de mayor significancia, siendo estas: la información y comunicación, las actividades de control, el ambiente de control y la evaluación de riesgos; todas ellas con un p-valor inferior a 0,05 y muy cercano a cero $(\mathrm{IC}=0.0000, \mathrm{ACC}=0.0053$, $\mathrm{AMC}=0.0001$ y ER $=0.0108$ ). Así también, en la misma tabla no se muestra la variable SM relacionada con la supervisión y monitoreo, todo ello debido a que por su bajo grado de significancia para el modelo ha sido excluida de él; y así también, porque al incluirla en las diferentes combinaciones realizadas de la ecuación, restaba significancia a las otras variables.

Tabla 3. Efectos marginales de la gestión del crédito en la probabilidad del impacto positivo de los factores de control interno

\begin{tabular}{|l|c|c|c|c|}
\hline \multicolumn{1}{|c|}{ Variable } & Coeficiente & Error estándar & Estadístico z & Probabilidad \\
\hline Información y comunicación & 1.342779 & 0.300418 & 4.469708 & 0.0000 \\
\hline Actividades de control & 1.302503 & 0.466666 & 2.791080 & 0.0053 \\
\hline Ambiente de control & 0.816712 & 0.213683 & 3.822081 & 0.0001 \\
\hline Evaluación de riesgos & 0.506864 & 0.198905 & 2.548270 & 0.0108 \\
\hline
\end{tabular}

Fuente: elaboración propia con base en los resultados arrojados por el software Eviews7. 
Cabe mencionar también que los resultados del modelo fueron tratados con la bondad de ajuste de Huber/ White para su corrección, opción que ofrece el Eviews para estimar de forma robusta la heteroscedasticidad de las variables; es así como el resultado del modelo presentó un $69.44 \%$ de aciertos de la bondad de ajuste, como lo muestra la tabla 4 .

Tabla 4. Porcentaje de aciertos del modelo

\begin{tabular}{|c|c|c|c|c|c|}
\hline \multicolumn{7}{|c|}{ Ecuación estimada } \\
\hline Valor & Observaciones & Correcto & Incorrecto & \% Correcto & \% Incorrecto \\
\hline 1 & 1 & 1 & 0 & 100.000 & 0.000 \\
\hline 2 & 3 & 1 & 2 & 33.333 & 66.667 \\
\hline 3 & 3 & 0 & 3 & 0.000 & 100.000 \\
\hline 4 & 49 & 35 & 14 & 71.429 & 28.571 \\
\hline 5 & 52 & 38 & 14 & 73.077 & 26.923 \\
\hline Total & 108 & 75 & 33 & 69.444 & 30.556 \\
\hline
\end{tabular}

Fuente: elaboración propia con base en los resultados arrojados por el software Eviews7.

Finalmente, la relación de los coeficientes de cada una de las variables en función de los puntos límites y considerando los valores promedios de ellas dio como resultado una probabilidad máxima del $55 \%$, como se muestra en la tabla 5 . En otras palabras, el modelo pronosticó que en la actualidad el 55\% de las cooperativas de ahorro y crédito del segmento 1 del Ecuador tiene buena gestión del crédito.

Tabla 5. Porcentaje de probabilidad máxima del modelo

\begin{tabular}{|c|c|c|c|}
\hline \multicolumn{4}{|c|}{ Con valores promedio } \\
\hline IC & ACC & AMC & ER \\
\hline $3,70 \mathrm{E}-07$ & $-3,34095 \mathrm{E}-18$ & $-2,7778 \mathrm{E}-07$ & $-3,7037 \mathrm{E}-07$ \\
\hline Incremental & & Probabilidad & Categoría \\
\hline $0 \%$ & & $0 \%$ & 1 \\
\hline $1 \%$ & $1 \%$ & 2 \\
\hline $1 \%$ & & $1 \%$ & 3 \\
\hline $57 \%$ & $55 \%$ & 4 \\
\hline $100 \%$ & & $100 \%$ & 5 \\
\hline Total & & & $55 \%$ \\
\hline \multicolumn{4}{|c|}{ Máxima probabilidad } \\
\hline
\end{tabular}

Fuente: elaboración propia con base en los resultados de la investigación. 
Por su parte, acorde con los resultados obtenidos en el modelo de Logit ordenado, se llegó a determinar cuáles de las variables de la hipótesis fueron aceptadas y cuáles rechazadas, en donde se presentan cinco hipótesis específicas planteadas en la presente investigación que poseen estadísticamente un resultado significativo (véase la tabla 3), es decir, todas ellas cuentan con valores inferiores al 0,5 de significancia, y así también sus coeficientes son relativamente altos; por lo que dichas hipótesis han sido asociadas a las interrelaciones del modelo, dando como resultado la consideración aceptable tanto teórica como práctica del modelo obtenido. Por otro lado, la hipótesis que establece que a mayor cumplimiento de los lineamientos de la supervisión y monitoreo se impacta positivamente en la gestión del crédito de las cooperativas de ahorro y crédito del segmento 1 del Ecuador fue rechazada. De donde resulta que la hipótesis general ha sido aceptada de la siguiente forma: el ambiente de control, la evaluación de riesgos, las actividades de control y la información y comunicación son factores que impactan positivamente en la gestión del crédito de las cooperativas de ahorro y crédito del segmento 1 del Ecuador.

\section{Conclusiones}

Los resultados obtenidos con el estudio realizado son la evidencia del análisis e identificación de los factores de control interno que impactan positivamente en la gestión del crédito de las cooperativas de ahorro y crédito del segmento 1 del Ecuador, siendo dichos factores el ambiente de control, la evaluación de riesgo, las actividades de control y la información y comunicación debido a que sus resultados estadísticos son significativos.

En este artículo se demuestra que los factores de control interno desempeñan un papel muy importante para predecir la probabilidad de que las coAc del segmento 1 del Ecuador cuenten con una buena gestión del crédito. En el periodo de estudio se encuentra que los factores de control interno relacionados con el ambiente de control, la evaluación de riesgo, las actividades de control y la información y comunicación impactan positiva y significativamente en la gestión del crédito. Asimismo se observa que el factor de control interno concerniente a la supervisión y monitoreo tiene un impacto no significativo en la gestión del crédito debido a que ya se encuentra inherente en la operatividad de los otros factores.

Desde el aspecto teórico, la presente investigación aporta considerablemente en demostrar que las cooperativas de ahorro y crédito del segmento 1 del Ecuador pueden fortalecer su gestión del crédito cumpliendo los lineamientos de los factores de control interno definidos en el modelo de Logit ordenado. Todo ello al considerar que las investigaciones en el tema cooperativo son reducidas, y mucho más en el ámbito relacionado con el control interno de la gestión del crédito, razón por la cual el presente estudio llega a constituir una importante contribución teórica específicamente en el ámbito académico, debido a que motiva a otros investigadores a continuar con la investigación. 
Por otro lado, desde el punto de vista práctico la investigación es relevante en el sentido que permite analizar e identificar los factores de control interno que impactan en el fortalecimiento de la gestión del crédito de las cooperativas de ahorro y crédito del segmento 1 del Ecuador; en razón de que este tipo de organizaciones presentan diferencias en su funcionamiento operativo y principalmente en su gestión, unas por desconocimiento funcional, otras por problemas en su sistema de control interno y otras por conductas inadecuadas de los que las dirigen; de esta manera, con los resultados obtenidos se determina el impacto significativo que tiene el ambiente de control, la evaluación de riesgos, las actividades de control y la información y comunicación, con aras al mejoramiento integral de este tipo de organizaciones y específicamente al fortalecimiento de su gestión de créditos. Lo cual es esencial para un eficiente sistema control interno, por lo tanto, para proporcionar un grado razonable a la compañía de alcanzar sus objetivos, en los aspectos que señala el informe coso (Orellana \& Gaete \& Gaete, 2002).

En este contexto, los hallazgos de este trabajo permiten sugerir ciertas estrategias de control interno que permitan hacer más eficiente la funcionalidad del proceso de gestión del crédito en las COAC del segmento 1 del Ecuador; considerando que la administración de las cooperativas dependerá del control que se realice, por eso el éxito o fracaso de una empresa cualquiera está estrechamente relacionado con una buena o mala administración, esto es más importante en las cooperativas por tener una organización y una vida económica encaminada a prestar buenos servicios a sus socios y a la comunidad en general (Trigo, 2012). Desde políticas inmediatas o de corto plazo, como la mejora en el servicio de otorgamiento del crédito específicamente en la estandarización del proceso a través de flujogramas y límites de tiempo definidos, así como en la unificación de requisitos y plazos de aprobación de créditos, hasta gestiones de largo plazo como políticas públicas de control interno orientadas al proceso de gestión del crédito en estas instituciones financieras. Para lograr lo anterior, las cooperativas de ahorro y crédito deben trabajar de manera conjunta con los respectivos entes de control tales como la Superintendencia de Economía Popular y Solidaria para llevar a cabo acciones que garanticen una seguridad razonable en dicho proceso.

Por consiguiente, el presente estudio no debe terminar con esta investigación, sino que debe considerarse un insumo para que los investigadores continúen con temas relacionados y profundicen sobre dichos aspectos, generando nuevas aportaciones tanto teóricas como prácticas que fortalezcan el sistema financiero popular y solidario, específicamente el sector cooperativo del Ecuador. 


\section{REFERENCIAS BIBLIOGRÁFICAS}

Ablan, N. y Z. E. Méndez (2010). Los sistemas de control interno en los entes descentralizados estadales y municipales desde la perspectiva coso. Visión Gerencial, 9 (1).

Alba, M. (2019). Editorial. Apuntes Contables, (23), 5-6. https://doi.org/10.18601/16577175. n23.01.

Betancourt, R. (2015). Impactos de la globalización en las cooperativas de ahorro y crédito en Ecuador. Ciencia Administrativa, (1), 123-130.

Castañeda, L. (2014). Los sistemas de control interno en las Mipymes y su impacto en la efectividad empresarial. En-Contexto Revista de Investigación en Administración, Contabilidad, Economia y Sociedad (2), 129-146.

Chaves, R., y J. L. Monzón (2001). Economía Social y Sector No Lucrativo: actualidad científica y perspectivas. CIRIEC-España, Revista de Economia Pública, Social y Cooperativa (37), 7-33.

Da Ros, G. (2007). El movimiento cooperativo en el Ecuador. Visión histórica, situación actual y perspectivas. Revista de Economía Pública y Cooperativa, 57, 249-284.

Dávila, R. (2003). Presente y Futuro del Cooperativismo Rural de Ahorro y Crédito en Colombia. Revista uniRcoop, vol. 1, n. ${ }^{\circ}$ 1, 140-153.

Díaz, M. P. M., \& Tubón, V. G. L. (2018). La evaluación del sistema del control interno como soporte estratégico en la gestión de objetivos en las finanzas populares del Ecuador. Apuntes Contables (21), 117-126.

Dorta, J. (2005). Teorías organizativas y los sistemas de control interno. Revista Internacional de Contabilidad \& Auditoría. Publicación Trimestral, abril-junio de 2005, 22, 9-58.

González, M. (2014). La importancia del control interno en las pymes.

Gutiérrez, N. (2009) Las Cooperativas de Ahorro y Crédito en el Ecuador y sus transformaciones durante los últimos diez años, Flacso Quito - Ecuador Maestría en Políticas Públicas.

Levin, A. y G. Verbeke (2002). Estado de la investigación sobre Cooperativa: Trabajo sobre el Cooperativismo argentino. CIRIEC-España, Revista de Economía Pública, Social y Cooperativa, 43, 23-39.

Mayorga Díaz, M. P. \& Llagua Tubón, V. G. (2018). La evaluación del sistema del control interno como soporte estratégico en la gestión de objetivos en las finanzas populares del Ecuador. Apuntes Contables (21), 117-126. https://doi.org/10.18601/16577175. n21.09.

Mejía, R. (2002). Sistema de control interno para las pequeñas y medianas empresas. Revista Universidad EAFIT, 38 (125), 74-86.

Miño, W. (2013). Historia del Cooperativismo en el Ecuador, Editogran S. A.

Orellana Flores, L., Gaete Araya, J. \& Gaete Becerra, H. (2002). Alternativas de valorizacion del sistema de control interno en las empresas. Chile. UChile.

Palacio, J. (2006). La investigación en materia de cooperativas de crédito y de grupos cooperativos. CIRIEC-España (56), 25-64. 
Pérez, César (2005), Métodos estadísticos avanzados con spss, International Thomson Editores, Madrid, España.

Saromo, S. y F. V. Pacheco (2014). El control interno y su influencia en la gestión de las empresas privadas de Latinoamérica, Perú y Chimbote, 2013, en Crescendo Ciencias Contables \& Administrativas, 1 (1).

Trigo Grey, O. H. (2012). El control interno como instrumento indispensable para la gestión de las Cooperativas de Ahorro y Crédito de Lima Metropolitana-periodo 1990-2010. Lima. Universidad de San Martín de Porres, repositorio academico.usmp.edu.pe.

Viloria, N. (2005) Factores que inciden en el sistema de control interno de una organización. Actualidad Contable FACES, año 8, n. ${ }^{\circ}$ 11. Mérida, Venezuela. 87-92. 